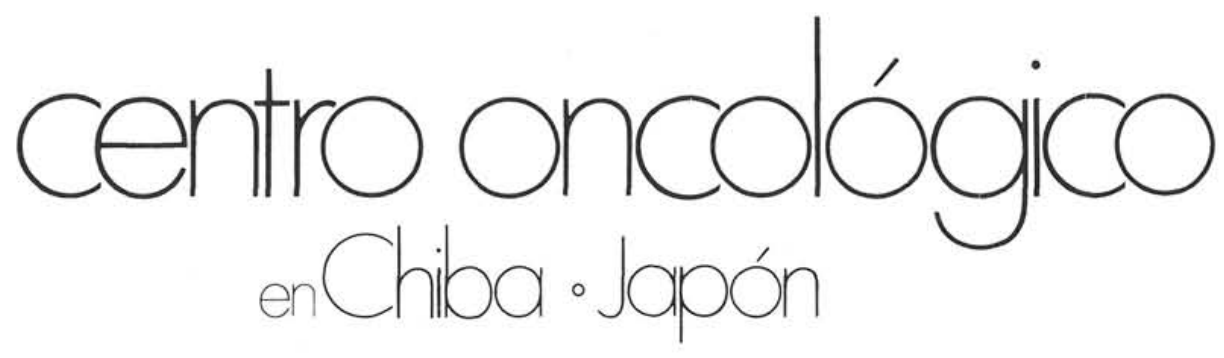

R. URA, N. NISHINO y M. ITOH, arquitectos

$143-64$

\section{sinopsis}

El edificio tiene capacidad para 200 camas -en la primera fase- y su proyecto consiste en un sistema de múltiples alas interrelacionadas que admiten la posibilidad de futuras ampliaciones, en línea o en bloque independiente, pero en conexión con el conjunto. En el primer caso se encuentran los cinco pabellones de: recepción de pacientes externos; radiografía y laboratorio de animales; fisiología, investigación y patología clínica; radioterapia; y cirugía y medicina nuclear. Los cuatro últimos tienen sótano y todos ellos una o dos plantas superiores. El segundo caso, de ampliación en edificio independiente, corresponde al pabellón de pacientes internos, el cual, con sus seis plantas más sótano, resulta el volumen más destacado del Centro. El bloque de administración, directamente relacionado con todos los demás, gracias a su posición central, está completamente definido, siendo el único cuerpo que no admite ampliaciones futuras.

Las grandes posibilidades del planteamiento arquitectónico, junto con los avanzados medios con los que está equipado, hacen de él uno de los más modernos y completos del mundo, entre los Centros de este tipo.

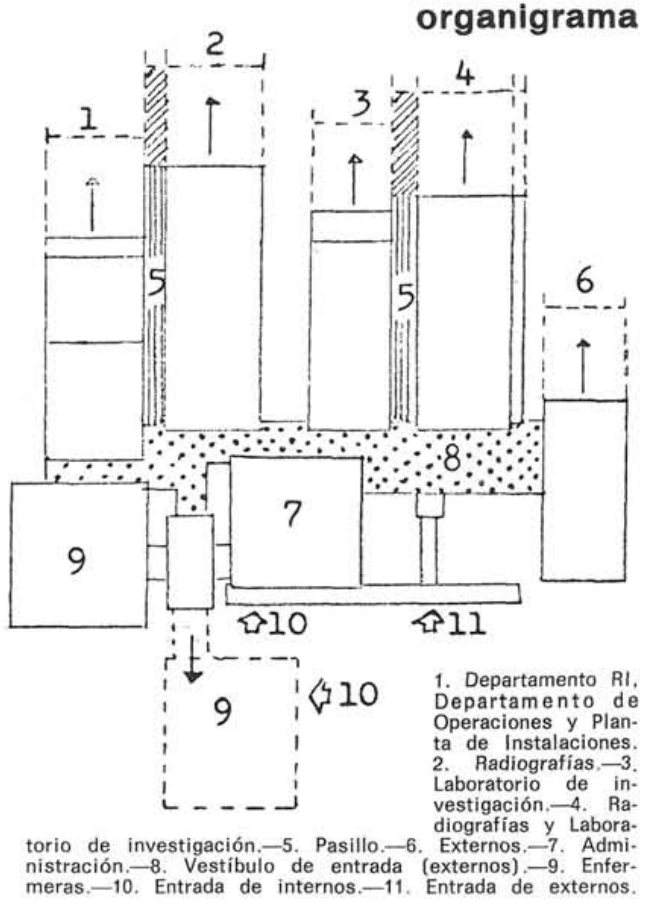

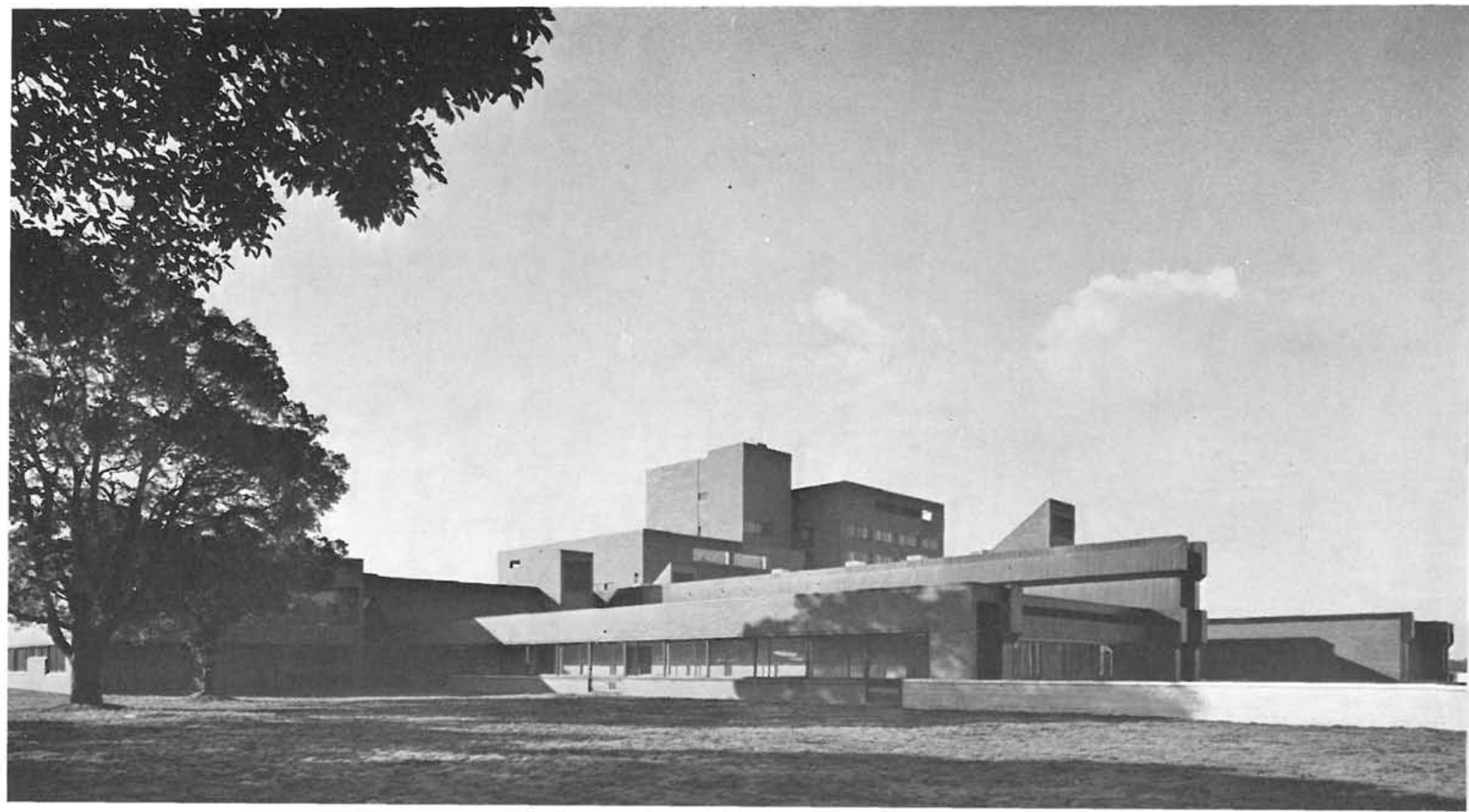




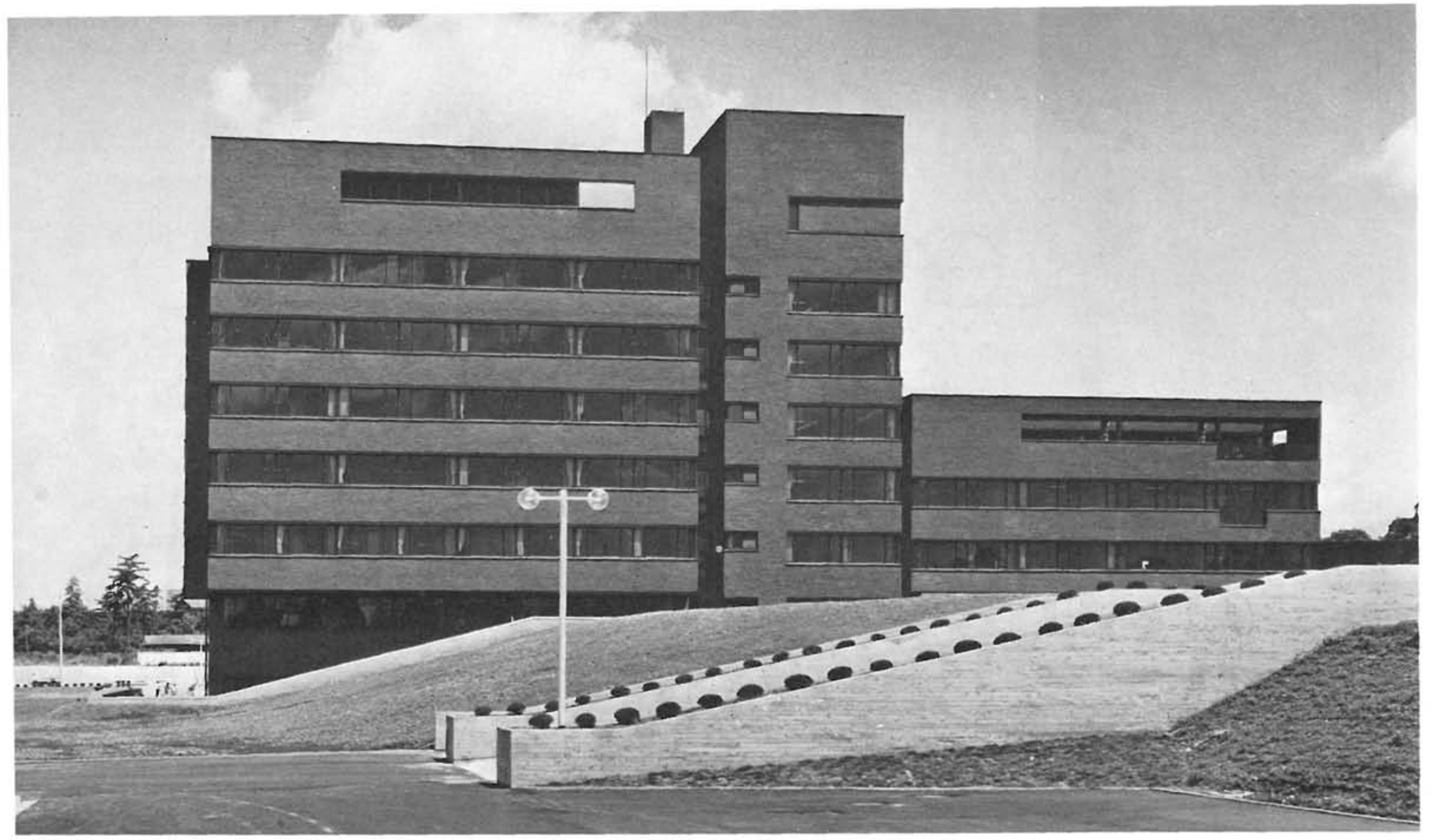

En la ciudad de Chiba, cerca de Tokyo, se ha construido un hospital con capacidad para unas 200 camas, destinado a Centro de Investigación para la lucha contra el cáncer. Este tipo de investigación se está llevando a cabo en todos los países del mundo, de forma más o menos intensa, utilizando hospitales gene- rales o instituciones expresamente dedicadas a la campaña, como ocurre en este caso.

Una de las más importantes premisas que condicionaron la planificación de este centro fue la forma de solucionar su previsible ampliación. Con una tecnología en pleno avance,

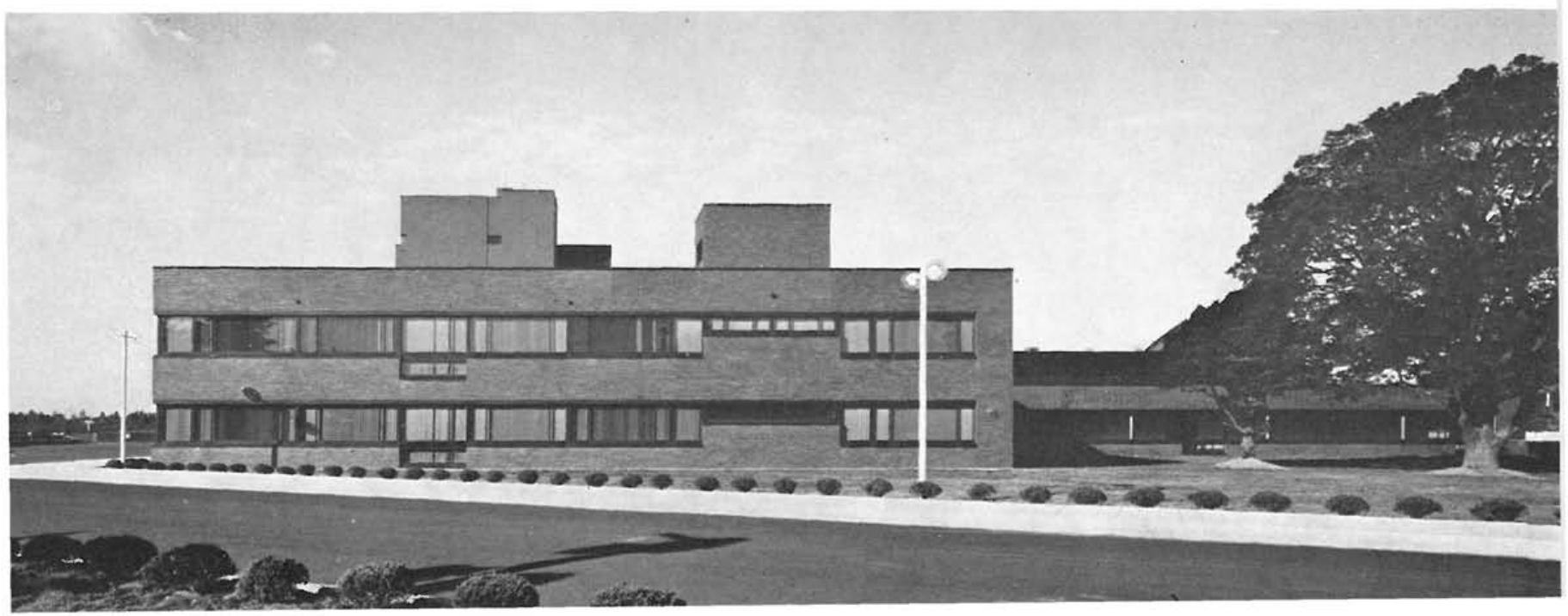



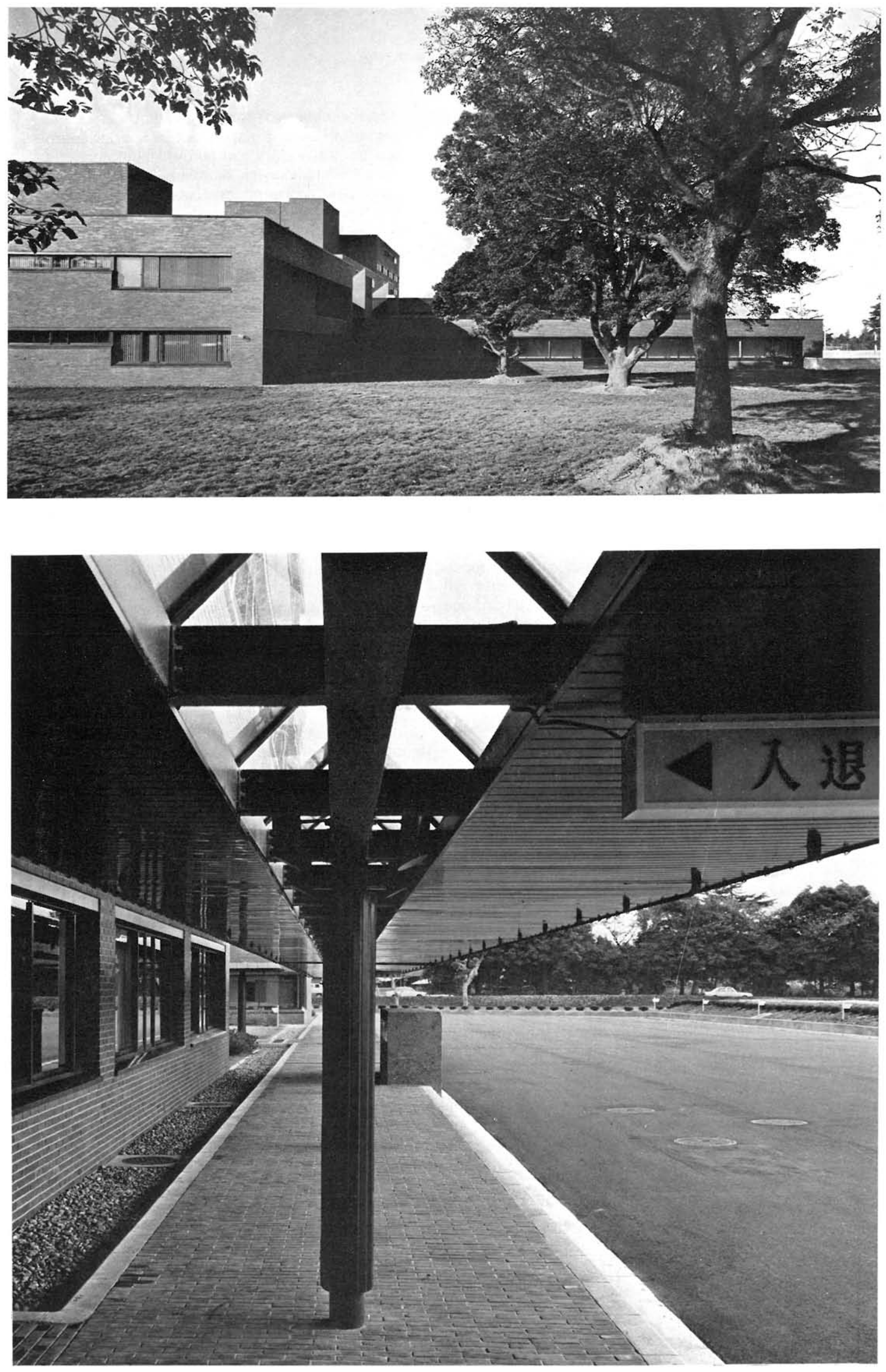
en la que en los últimos años ha entrado a formar parte la energía nuclear, era lógico suponer cambios importantes en los medios y en sus respectivas aplicaciones y formas de utilización, y, por tanto, en sus necesidades de espacio. Por ello, al realizar el diseño de este proyecto fue previsto un sistema de planificación pensado en el futuro, de tal forma que hiciera posible adaptar el edificio original a cualquier cambio motivado por el desarrollo.

De acuerdo con lo anterior, el hospital se diseñó según un plan de múltiples alas, o cuerpos diferenciados, unidos en cada nivel mediante pasillos y terrazas. Cada departamento del Centro ocupa zonas de estas alas, constituyendo pabellones independientes pero agrupados en el conjunto, y que dejan uno de los extremos dispuesto para una posible ampliación independiente del resto.

Entre cada dos alas se ha dispuesto una galería o pasillo que sirve de acceso a los distintos locales, y que, del mismo modo, puede participar en el proceso de crecimiento del conjunto. Los bloques tienen una anchura que oscila entre 15 y $18 \mathrm{~m}$, dimensión que se adapta perfectamente el tipo de estructura más adecuada para estos centros.

\section{ORGANIZACION DEL CENTRO}

Los enfermos que acuden al hospital para ser examinados penetran a un hall, comunicado directamente con el pabellón de recepción de pacientes, y con un pasillo, a modo de espacio central, en el que desembocan las galerías de distribución. También los pacientes internos cuentan con su propia entrada, conectada de igual forma con el espacio central y con un nudo independiente de comunicaciones verticales, sirviendo al mismo tiempo de acceso para el pabellón de administración. Estas dos entradas se emplazaron en la primera planta del conjunto, mientras que una tercera, para servicios, se dispuso en un nivel inferior.

El pabellón de recepción de pacientes está constituido por un bloque de dos plantas, sin sótano, en el que se concentran todos los equipos necesarios para realizar un primer examen al paciente en toda una amplia gama de especialidades médicas relacionadas con el cáncer.
Anexo a este pabellón se encuentra el de radiografía, de una sola planta superior, pero con un sótano que contiene el laboratorio de experimentación con animales y que incluye las dependencias necesarias para cría y mantenimiento de los mismos.

Una de las galerías principales sirve de separación entre el pabellón anterior y un cuerpo de dos plantas, más sótano, en los cuales se distribuyen el laboratorio de fisiología, un completo departamento de investigación y el laboratorio de patología clínica.

En el otro extremo del edificio, el pabellón de pacientes internos constituye el volumen más destacado del conjunto. Consta de seis plantas y un sótano, donde se sitúan los departamentos de servicios, la sala de autopsias y el depósito de cadáveres. En las plantas superiores se han distribuido la totalidad de las habitaciones para los pacientes, así como los servicios complementarios para uso de las enfermeras.

Junto a este último cuerpo se encuentra el ala dedicada a cirugía, con la que está relacionado de forma muy directa.

En su primera planta se colocaron las salas de quirófanos y sus correspondientes locales auxiliares, mientras que en la segunda se desarrolla el departamento de medicina nuclear. El sótano se ha aprovechado para instalar la planta técnica que abastece de energía a todo el conjunto.

Un segundo pasillo principal separa el pabellón de cirugía de otro, de una sola planta, en el que se sitúa el departamento de radioterapia.

Por último, el pabellón de administración, emplazado en fachada y en la zona central, está formado por un bloque de cuatro plantas, más un sótano, en el cual se ha organizado un departamento de servicios, complementario del existente en el pabellón de pacientes internos. En el resto de las plantas alternan los despachos y oficinas administrativas, con secciones de farmacia, biblioteca, salas de lectura, comedores, y con el centro de computadoras.

En el sistema de planificación con el que se ha desarrollado el Hospital, figura el pabellón administrativo como una unidad fija, mientras que los demás pabellones permiten una posi- 


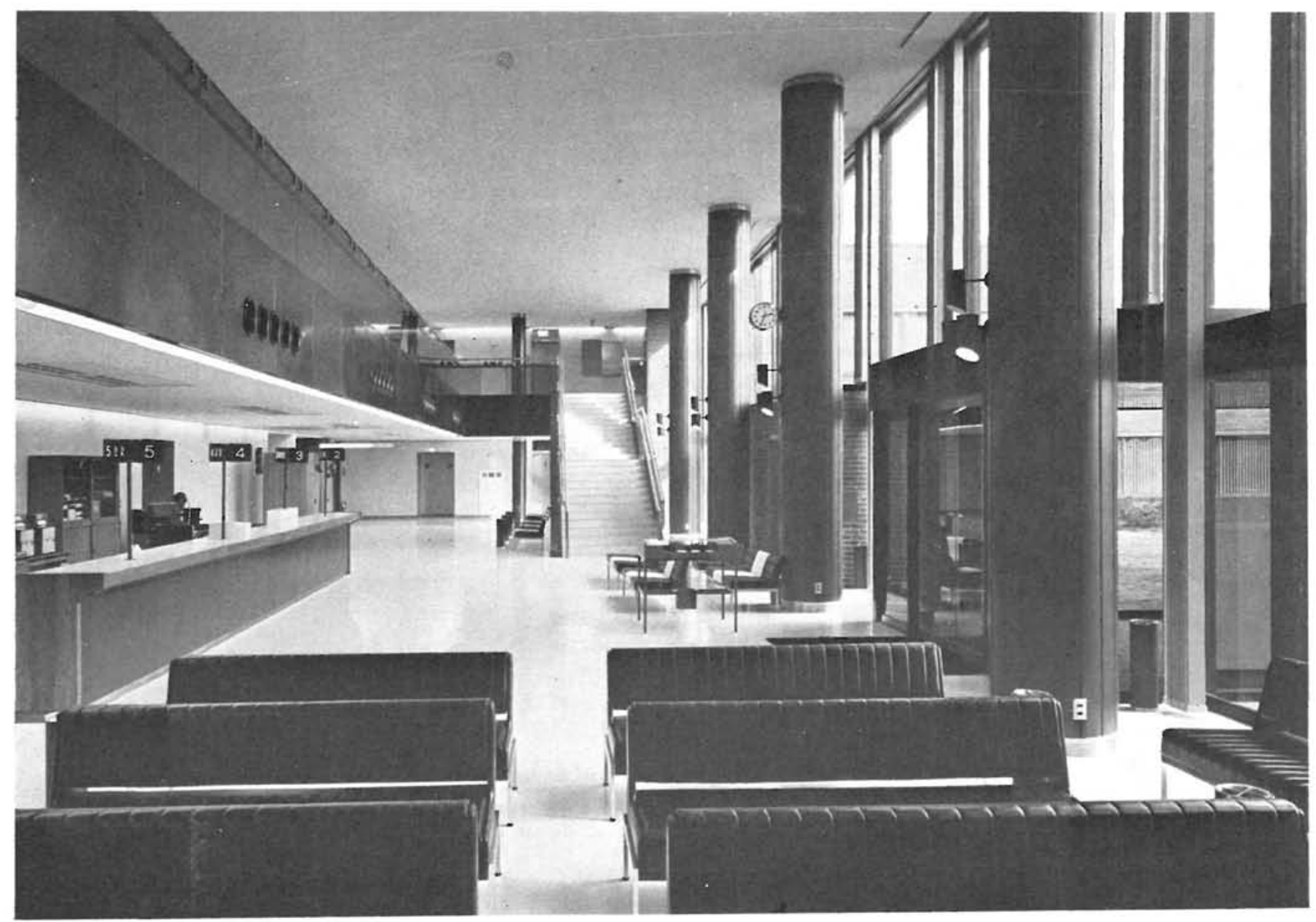

A. O.P.D.

B. Laboratorio de animales $\mathrm{x}$ rayos

C. Investigación, fisiología y patología clinica.

D. Instalaciones eléctricas

E. Sala de máquinas, operaciones $y$ medicina

Servicios $y$

G. Servicios, Administración, lectura, com y ordenadores. Entrada de Entrada

Entrada de

c. Patio.

planta general

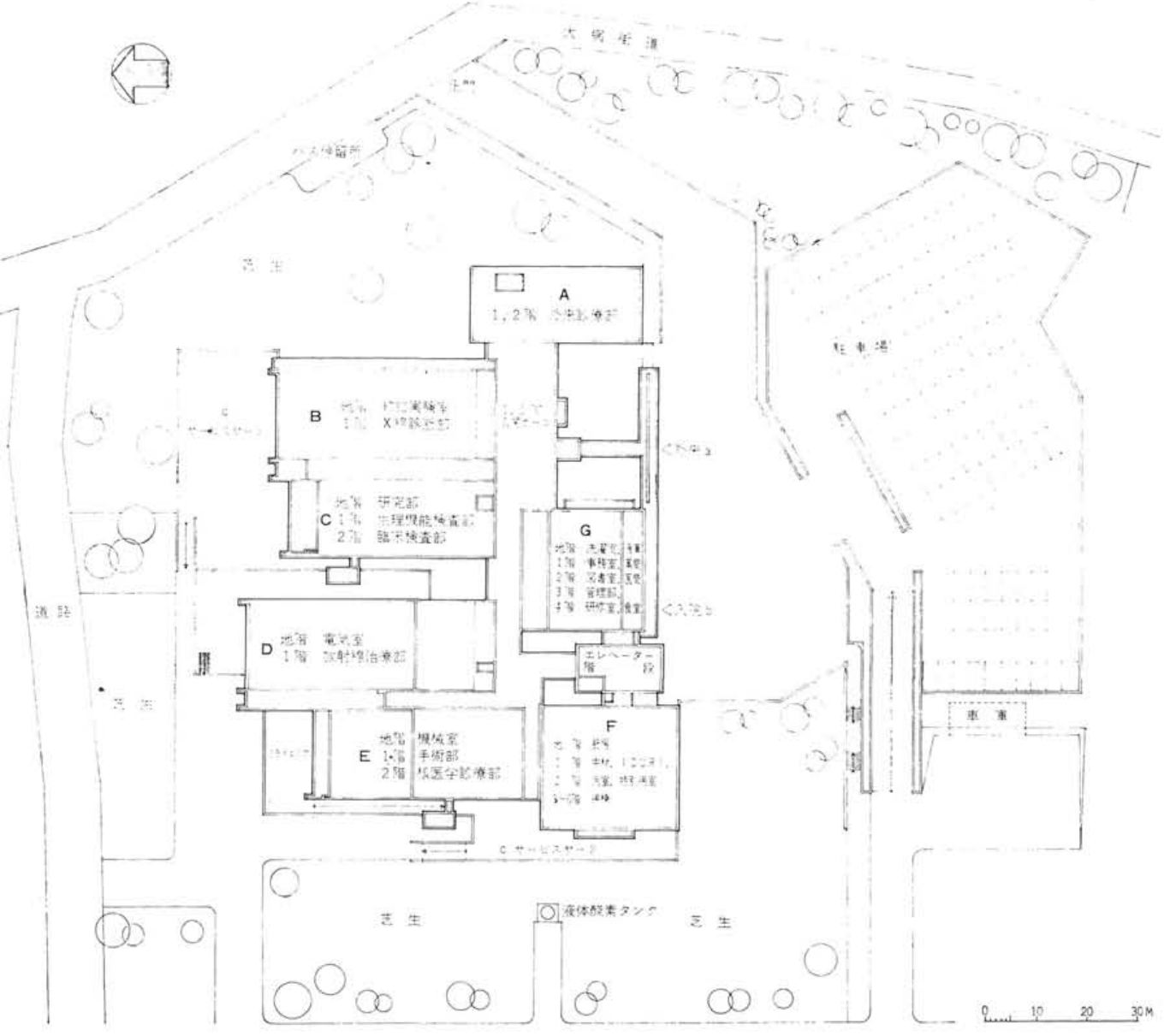

配 置図 


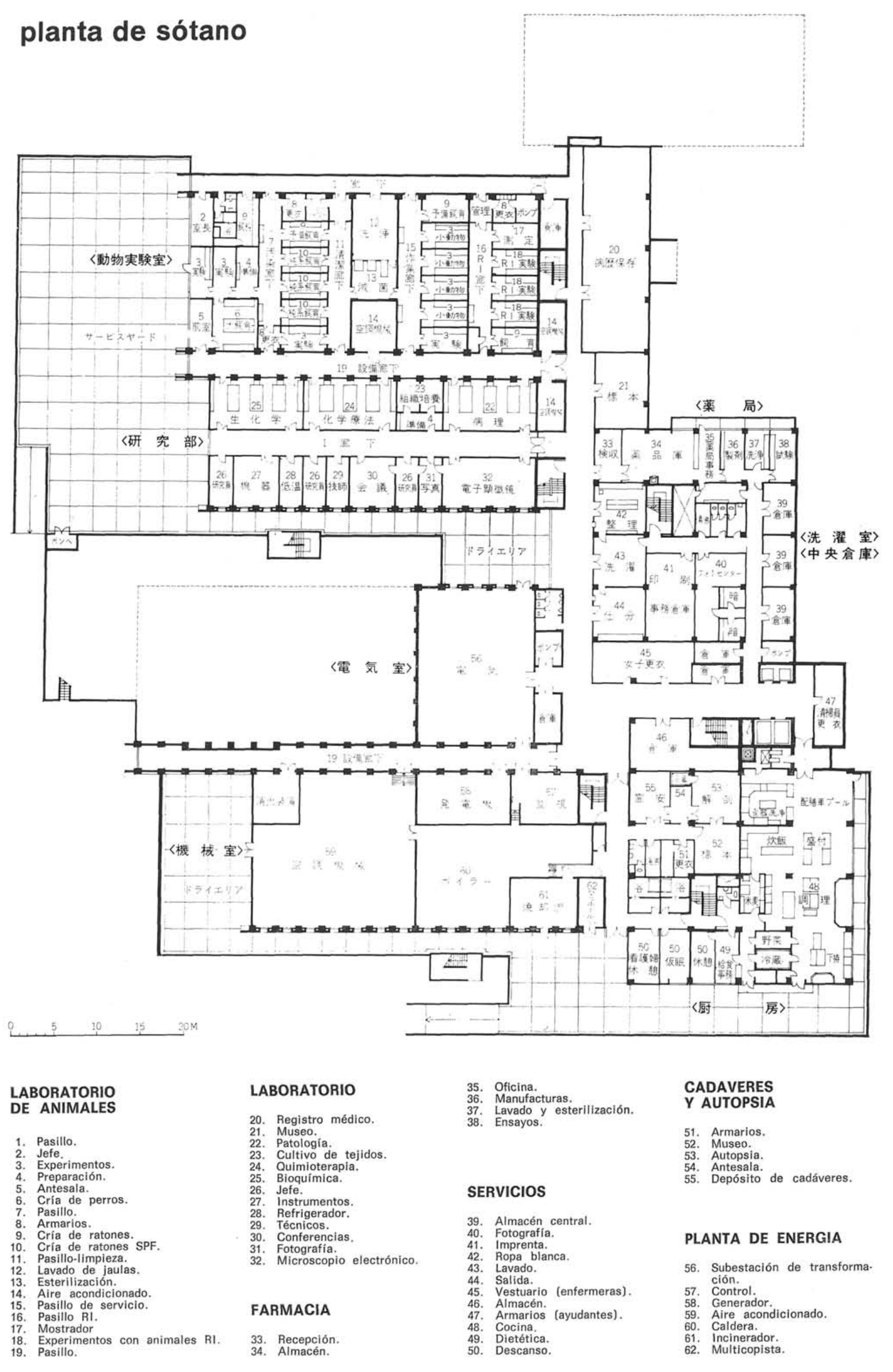


planta primera

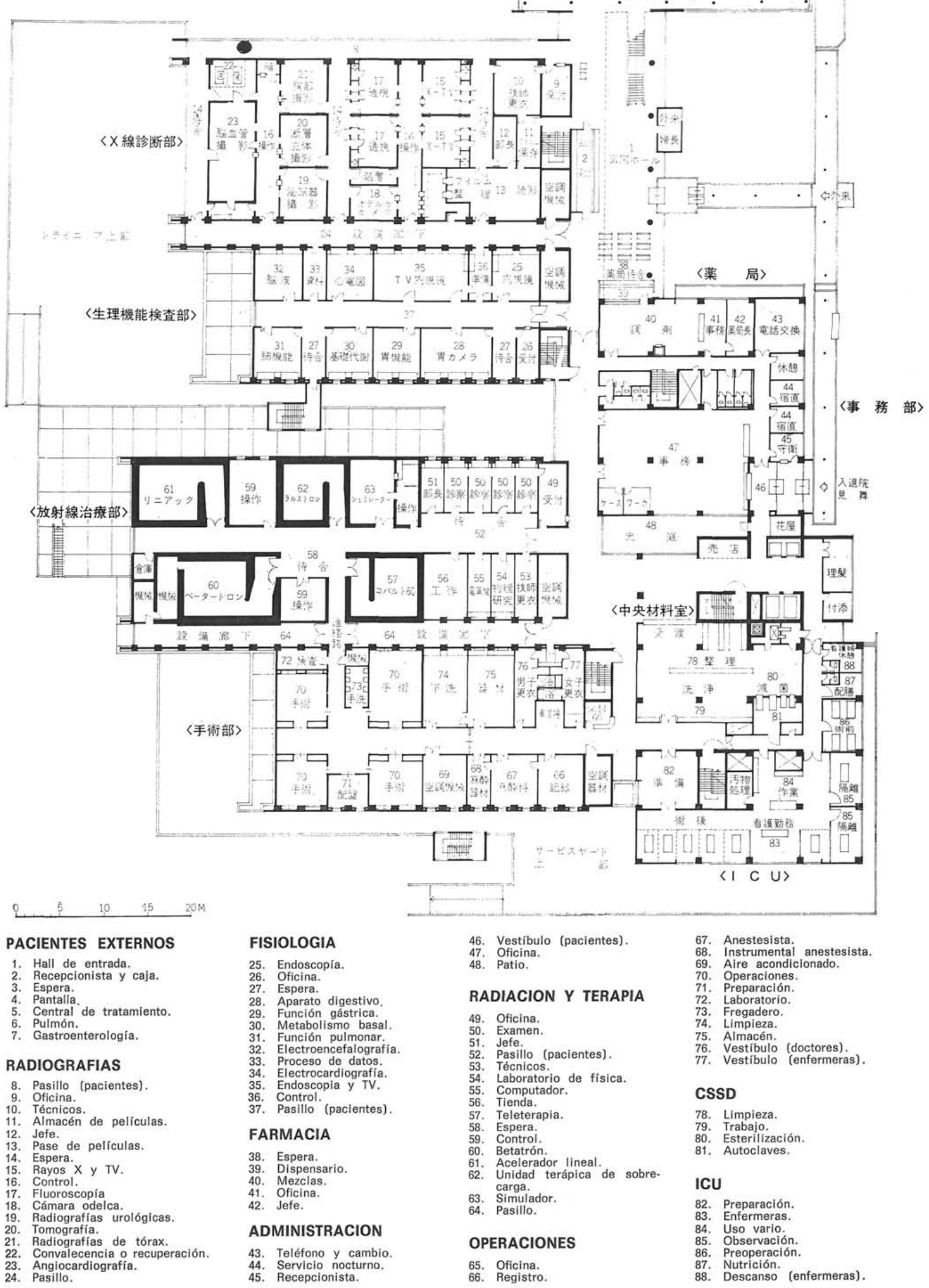




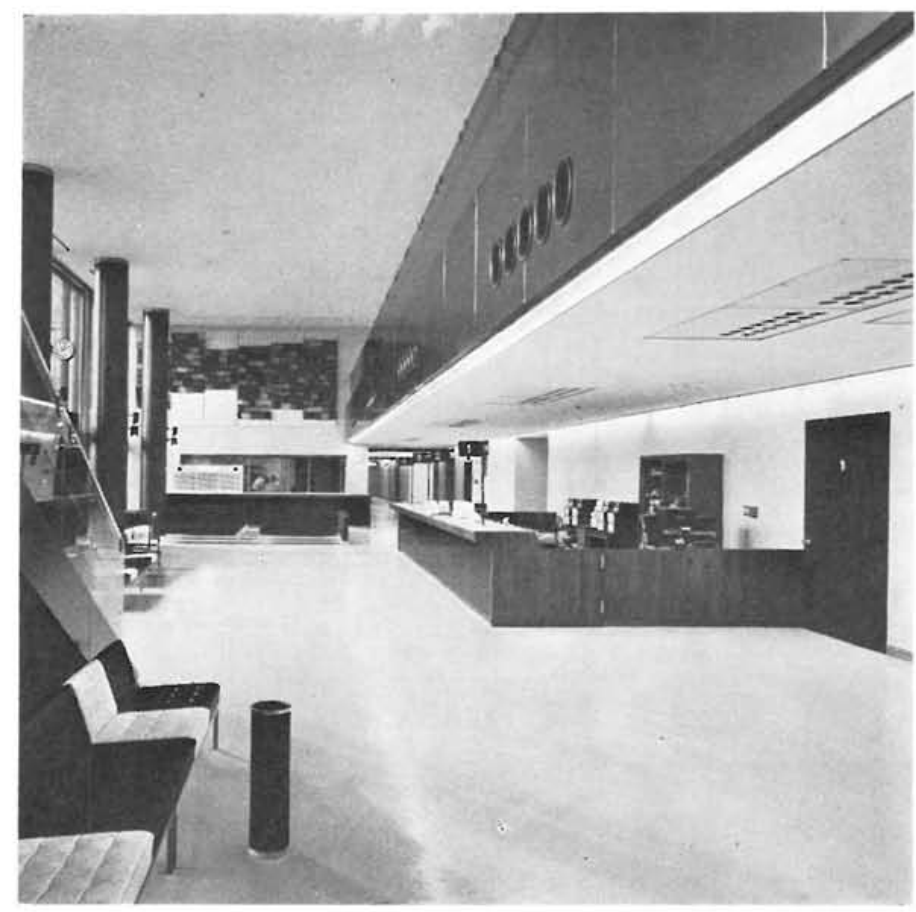

\section{PACIENTES EXTERNOS}

1. Examen de ginecologia.-2. Examen de urologia y dermatologia. - 3. Examen de otorrinolaringología.-

\section{CLINICA PATOLOGICA Y LABORATORIO}

7. Pasillo.-8. Técnicos.-9. Registro--10. Instrumentos. 11. Laboratorio,-12. Probetas - 13. Oficina,-14. Jefe.17. Microbiología.

\section{ADMINISTRACION}

18. Descanso.-19. Doctores.-20. Biblioteca.-21. Epide miologia.-22. Computador.

PRIVADO, DORMITORIO DE ENFERMERAS

\section{$Y$ R}

23. Acompañante.-24. Descanso (enfermeras).-25. Despensa.-26. Dormitorio privado-27. Dormitorio RI. 31. Enfermeras.

\section{MEDICINA NUCLEAR}

32. Oficina.-33. Espera.-34. Aire acondicionado.-35 Tiroides, - 36. Riñón.-37. Escintigrama - -38. Examen y tratamiento.-39. Laboratorio de química.-40. Preparacontaminación. - 43. Control.

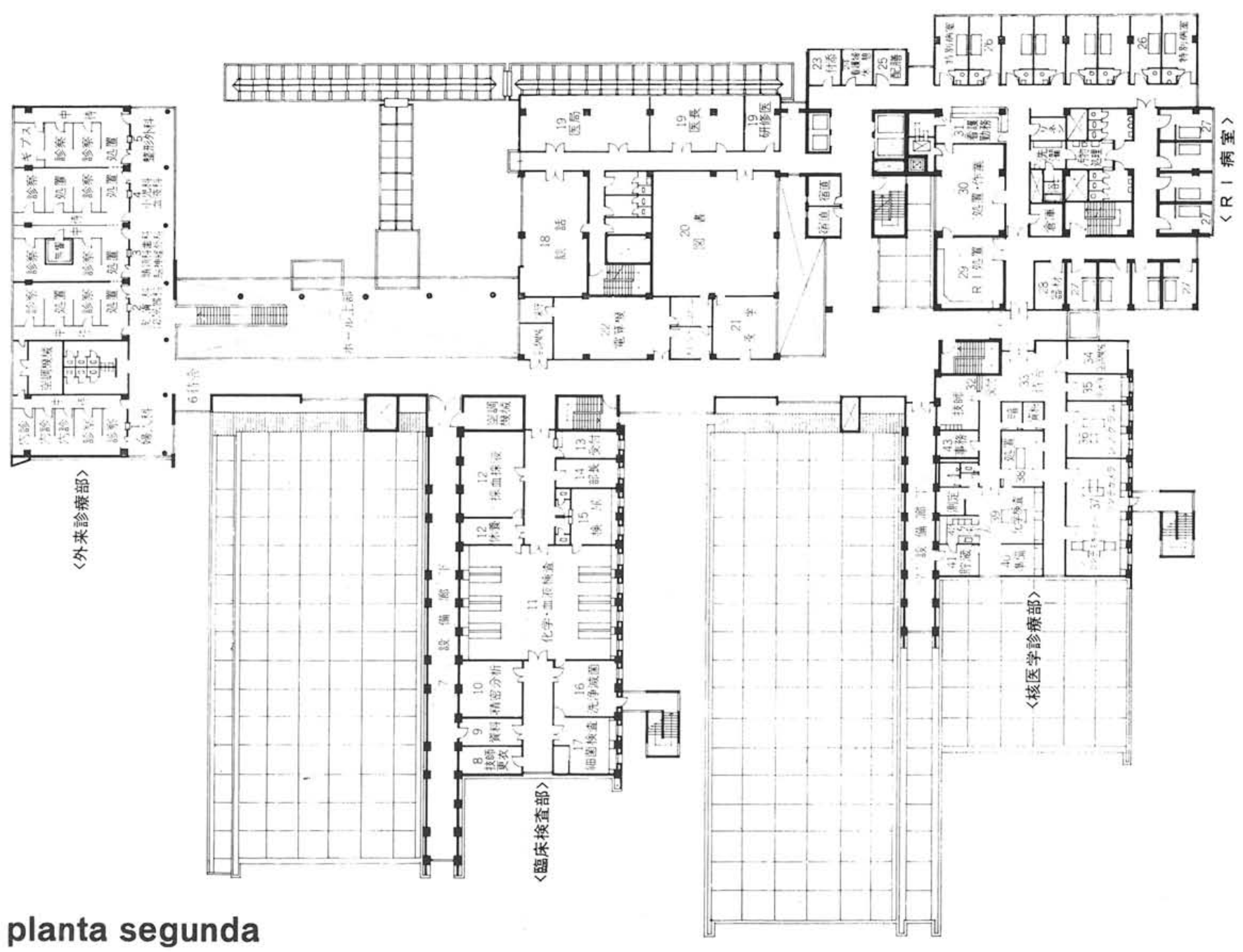




\section{planta tercera}

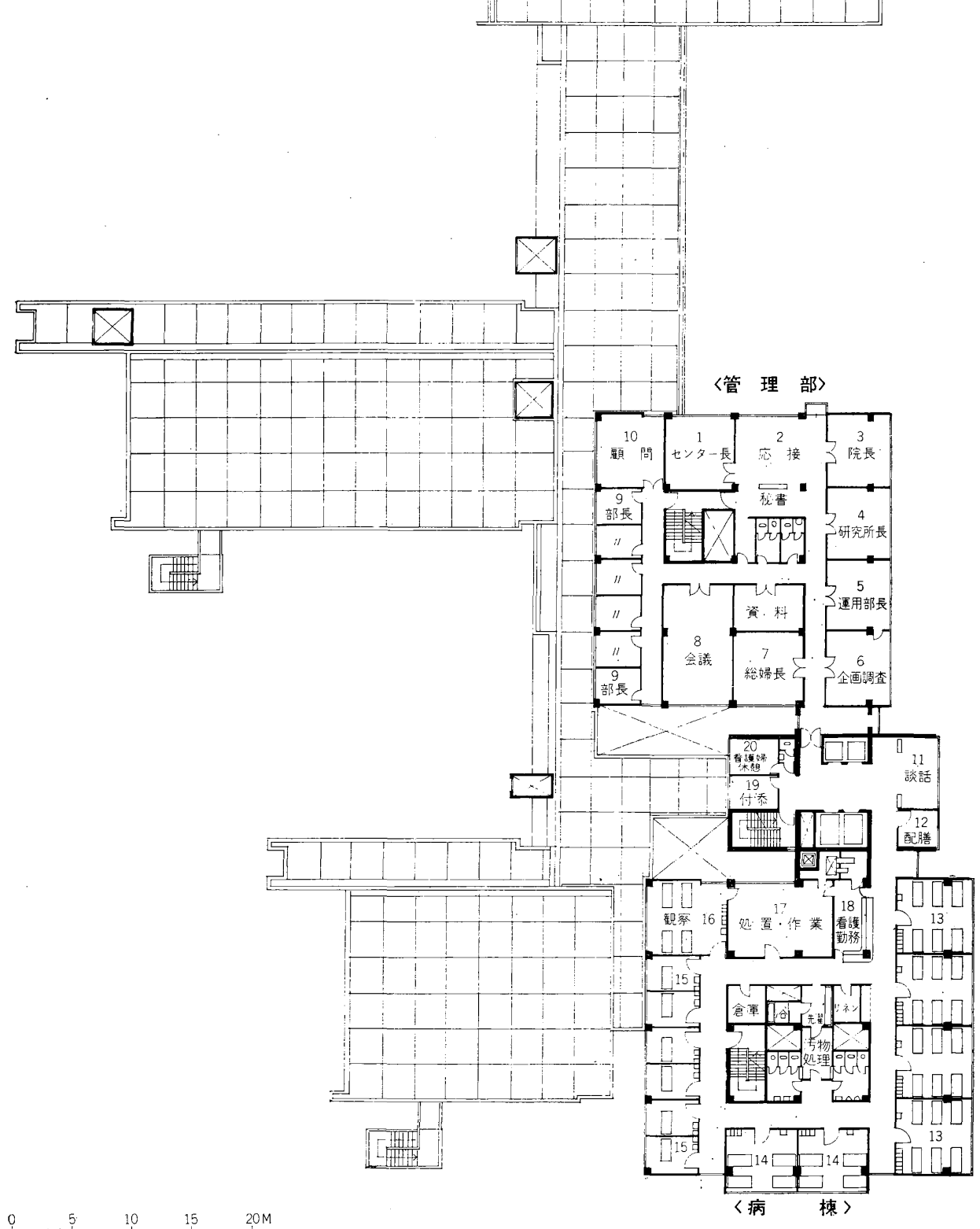

\section{ADMINISTRACION}

1. Administrador generai.

Descanso (dirección).

Director del Hospital.

Director del Labor

Administrador.

Despacho de investigacion.

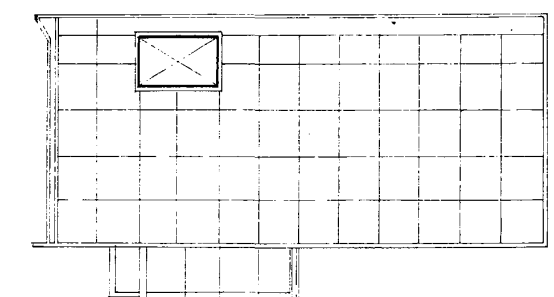

〈管 理 部〉
8. Conferencias.
9. Doctor jefe.
ENFERMERAS
11. Descanso (pacientes).
12. Despensa.

Habitación (6 camas)

14. Habitación (4 camas)

15. Habitación (1 cama).

16. Cama de observación.

17. Uso vario.

18. Enfermeras.

20. Descanso (enfermeras). 


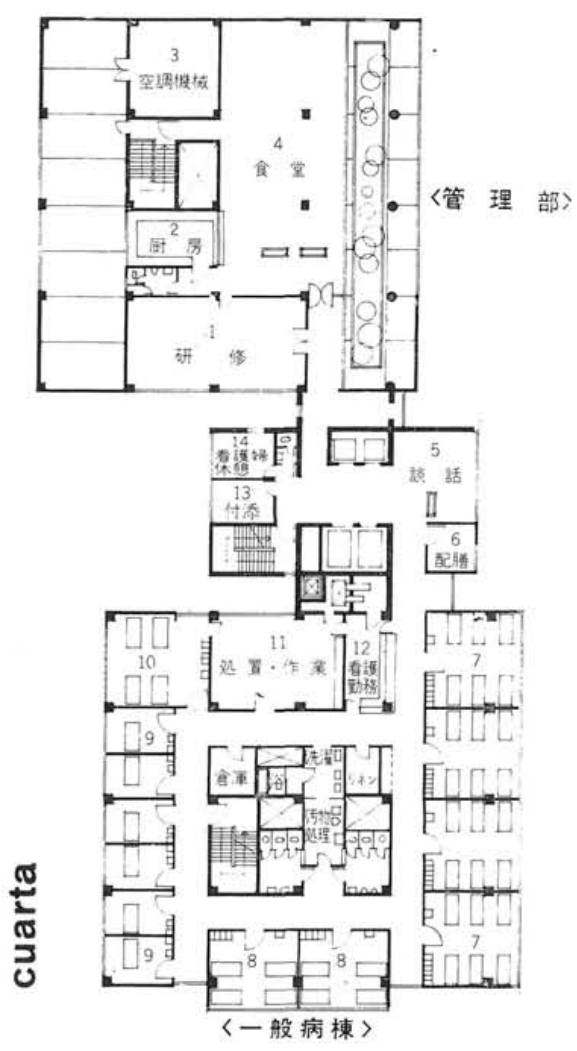

\begin{abstract}
plantas

\section{ADMINISTRACION}

1. Lectura y conferencias. -2 . Cocina. -3 . Aire acondicionado.-4. Comedor (dirección).

\section{ENFERMERAS}

5. Descanso (pacientes) - 6. Despensa.-7. Habitación (6 camas). - 8. Habitación (4 camas). -9. Habitación (1 cama). -10. Cama de observación.-11. Uso vario.
\end{abstract}

\title{
ULTIMA PLANTA
}

1. Maquinaria ascensor.-2. Aire acondicionado.
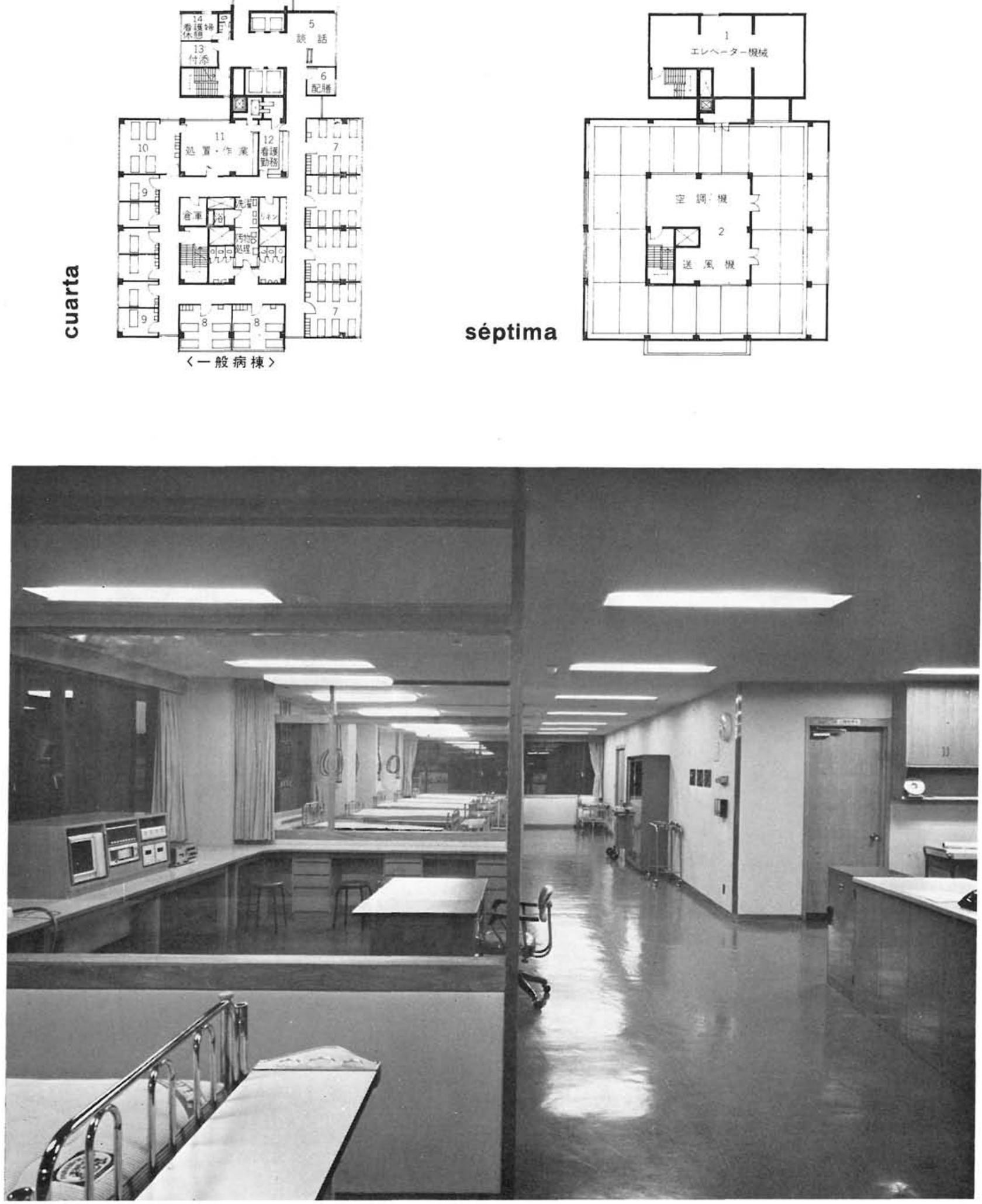

(C) Consejo Superior de Investigaciones Científicas Licencia Creative Commons 3.0 España (by-nc) 

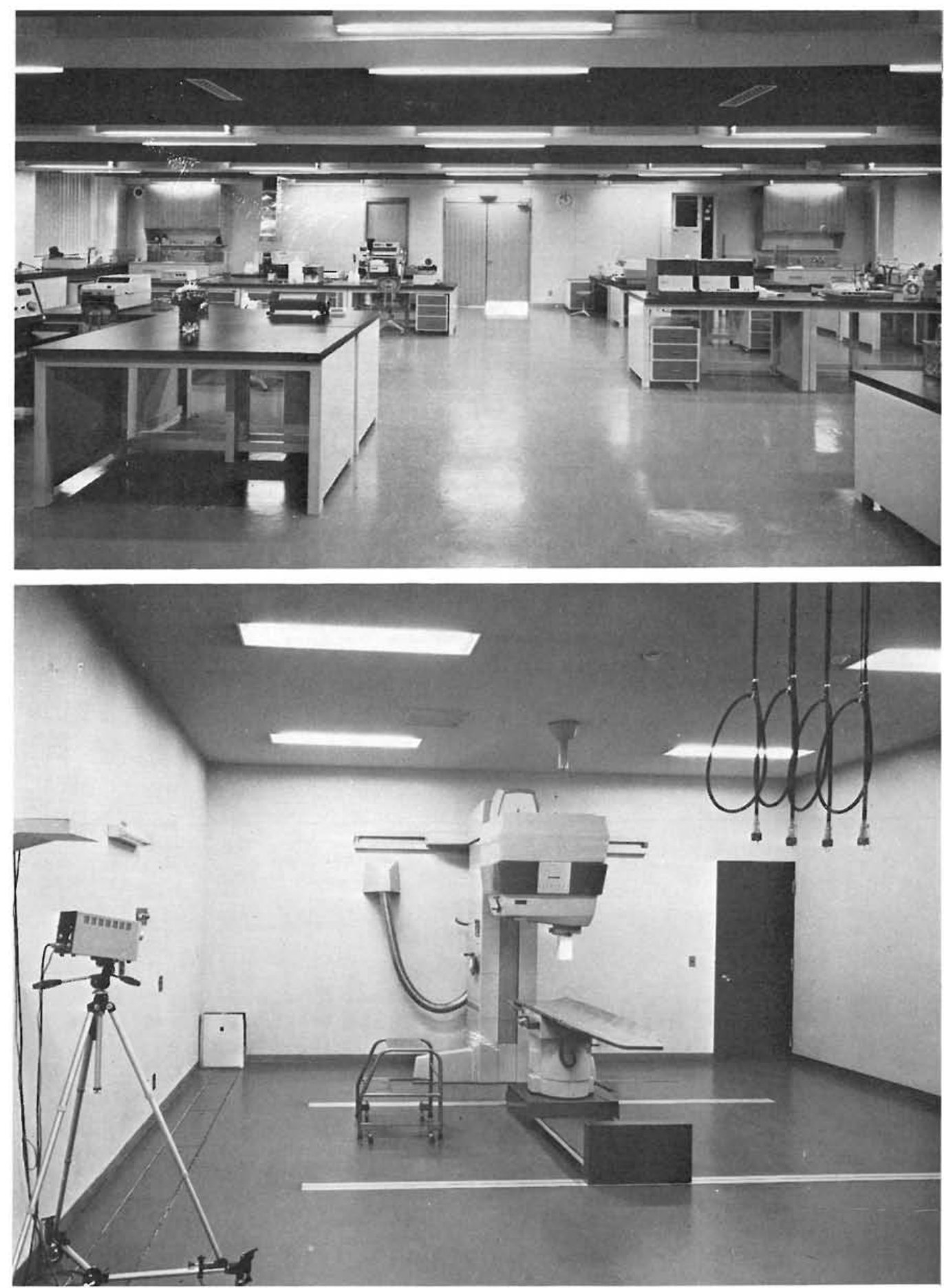

FOTOS: KAWASUMI

ble ampliación. El pabellón de pacientes internos tiene prevista esta posibilidad por medio de un bloque independiente que se situaría anexo y conectado al conjunto. Los demás bloques o cuerpos podrán ampliarse desarrollando linealmente sus estructuras originales.

Con estas posibilidades futuras y su realidad presente, este Centro hospitalario de investigación para la lucha contra el cáncer se encuentra entre los más modernos y mejor equipados del mundo. 


\section{résumé}

Centre oncologique à Chiba - Tokyo Japon

R. Ura, N. Nishino et M. Itoh, architectes

Le bâtiment peut abriter 200 lits -dans la première phase - et son projet consiste en un système d'ailes multiples reliées entre elles, susceptibles d'etre agrandies dans mais' en liaison avec l'ensemble. Dans Ie premier cas se trouvent les cinq pavillons premier cas se trouvent les cinq pavillons de: réception de patients externes; radiographie et laboratoire d'animaux; physiologie, recherche et pathologie clinique; radioLes quatre derniers out un sous-sol et tout les pavillons ont un ou deux niveaux supé. rieurs. Le second cas, d'agrandissement en immeuble indépendant, correspond au pavillon de patients internes, qui, avec ses six niveaux avec sous-sol, est le volume le plus remarquable de ce Centre. Le bloc d'administration, directement uni à tous les autres grâce à sa position centrale, est completement défini, étant le seul corps qui n'admet pas d'agrandissements futurs.

Les grandes possibilités du parti architectural, ainsi que les moyens avancés dont il est equipe, font de ce batiment lun des plus modernes et complets

\section{summary}

Cancer Clinic in Chiba - Tokyo Japan

R. Ura, N. Nishino and M. Itoh, architects

In its first phase this hospital disposes of 200 beds and the project consists of which provides for future entargent either in a row or in an independent block, which however, should be connected to the rest of the complex. In the first mentioned case the five buildings contain: receptio of out-patients; X-ray department and anima laboratory; physiology, research and clinic pathology; radiotherapy; surgery and nuclear medicine. The latter four all have a basement and one or two storeys. In the second case, the independent block would be for in-patients and having 6 storeys plus basement it will be the most prominen tion block is situated in the admistra directly connected with all the other blocks. This is the only one that has been contructed in its final form and has been contructed enlarged.

As a result of the great architectural possi bilities and the highly advanced equipment this is and the highly advanced equipment plete hospitals of its kind in the world.

\section{zusammenfassung}

Cancer Clinic in Chiba - Tokyo Japan

R. Ura, N. Nishisno und M. Itoh,

Das Krankenhaus verfügt in seiner ersten hase über 200 Betten und der Entwurf der verbundenen Flügeln dar, wodurch zukünftiger Ausbau, antweder in einer Linie oder in einem unabhängigen, jedoch mit den übrigen Bestandteilen der Klinik in Verbindung stehenden Block, ermöglicht wird. In dem ersten Fall umfassen die fünf Gebaude folgendes: Empfang auswärtiger Patienten; Rontgenabteilung und TierlaboraPrium; Physiologie, Forschung und klinische Kernmedizin. Die letzten vier bestehen aus Kellergeschoss und einer oder zwei Etagen. Die zweite Alternative - Ausbau in unabhängigem Block - ist ein Gebäude für Internpatienten, welches mit seinen sechs Etagen und Kellergeschoss das grösste dieses Zentrums sein würde. Der in der Mitte gelegene und mit den übrigen Komponenten direkt der in seiner endgültigen Form erbaut worden ist und dadurch nicht ausgebaut werden kann.

Zufolge der grossen architektonischen Möglichkeiten, neben der hervorragenden Ausmodernsten dieser Art auf der Welt.

\section{publicación del i. e. t. c.e.}

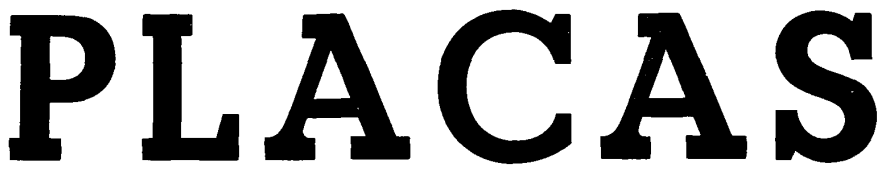

\section{K. Striglat in H. Wippel}

Drs. Ingenieros

Traducción de Juan Batanero Dr. Ingeniero de Caminos

con la colaboración de

Francisco Morán

Ingeniero de Caminos

Este libro, cuidadosa y magníficamente editado, reúne, quizás, la más completa colección conocida de tablas para placas, por los numerosos casos de vinculación y de carga estudiados y por la abundancia de relaciones de dimensión y de datos ofrecidos, que cubren prácticamente todo el campo de las losas en edificación. Permite desarrollar, con comodidad, rapidez y una aproximación suficiente, los cálculos de dimensionamiento y comprobación, obviando las dificultades que como es sabido, presenta el desarrollo numérico de los métodos de cálculo de estos elementos, evitando enojosas operaciones.

Trata la obra sobre "Zonas de Placas», "Placas sobre apoyos puntuales», "Placas apoyadas en dos tres y cuatro bordes" y «Placas apoyadas elásticamente», tipos que en la actualidad disponían de una documentación, incompleta o nula, para la determinación de esfuerzos. Los corrimientos de la placa, como valores previos para la determinación de los momentos, han sido obtenidos por medio del Cálculo de Diferencias, método que se ha comprobado como suficientemente satisfactorio, aún en su forma simple, aplicado con un cierto control.

Un volumen encuadernado en tela, de $30,5 \times 23,5 \mathrm{~cm}$, compuesto de 92 págs. Madrid, 1968.

Precios: España, 925 ptas.; extranjero, \$18.50. 